\section{( OPEN ACCESS}

\title{
Cross-sectional study on the prevalence of HPV antibodies in the general population of the Czech Republic
}

\author{
Eva Hamsikova, Viera Ludvikova, Jana Stasikova, Ruth Tachezy
}

Department of Experimental Virology, Institute of Hematology and Blood Transfusion, Praha 2, Czech Republic

\section{Correspondence to} Dr Eva Hamsikova, Department of Experimental Virology, Institute of Hematology and Blood Transfusion, U Nemocnice 1, 12820 Praha 2, Czech Republic;

eva.hamsikova@uhkt.cz

Accepted 9 May 2012 Published Online First 8 June 2012

\begin{abstract}
Objectives The assessment of the prevalence of antibodies to human papillomaviruses (HPV) in the healthy population is essential for effective planning of HPV vaccine implementation into the preventive programmes for HPV-associated diseases and for the prospective monitoring of the impact of HPV vaccines in the Czech population.
\end{abstract}

Methods The seropositivity for HPV-6, 11, 16, 18, 31 and 33 virus-like particles was determined in sera from 3150 healthy individuals (age range $6-76$ years) by means of enzyme-linked immunoassay.

Results The seroprevalences for HPV-6, 11, 16, 18, 31 and 33 were $23.8 \%, 15.2 \%, 14.5 \%, 9.9 \%, 16.4 \%$ and $9.6 \%$ in women and $18.4 \%, 13.7 \%, 6.5 \%, 5.4 \%, 6.1 \%$ and $4.3 \%$ in men. For both genders, except for HPV11, these rates were age dependent. The prevalence of antibodies to HPV-16 and/or 18 reached the maximum of $27.0 \%$ in women $30-39$ years of age and of $14.4 \%$ in men 50-59 years of age. The highest proportion of individuals' seropositive for any of the vaccine types HPV-6/11/16/18 was in 30- to 39-year-old women $(50.0 \%)$ and in $\geq 60$-year-old men (37.6\%). Antibodies specific for vaccine HPV types were detected in $18.0 \%$ of children 6 - to 14 -year-old but in $26.4 \%$, those older than 14 years.

Conclusions The data reveal age-specific differences in the HPV seropositivity rates between healthy women and men and support the implementation of HPV vaccination in the Czech Republic before the age of 13 .

\section{INTRODUCTION}

Cervical cancer is the third most common female cancer worldwide with estimated 530000 incident cases and about 275000 deaths in $2008 .^{1}$ There is a substantial molecular, biological, epidemiological and immunological evidence for the etiological role of oncogenic (high-risk, HR) types of human papillomaviruses (HPV) in the development of cervical cancer and the respective precursor lesions. ${ }^{2}$ HPV-16 and -18 are the most common oncogenic types which account for approximately $70 \%$ of cervical cancer cases worldwide. ${ }^{3}$ The same HPV types are also considered as a possible etiological factor of other anogenital dysplasias and cancers, that is, dysplasias and cancers of the vulva, vagina, penis, anus and oropharynx. ${ }^{4}$ Low-risk (LR) HPV types, namely HPV-6 and -11, are involved in the development of genital warts and recurrent respiratory papillomatosis. ${ }^{5}$ Nowadays, HPV infection is the most common sexually transmitted viral infection in the developed countries; more than $50 \%$ of sexually active individuals are infected during their lifetime. Following natural infection, antibodies specific to HPV capsid proteins are expanded; however, it is a slow process, which does not necessarily occur in every woman. ${ }^{6}$ It has been shown recently that long-lasting high HPV-specific antibody levels following natural infection are associated with reduced risk of subsequent infection with concordant as well as with closely phylogenetically related HPV type. ${ }^{7}$

The detection of HPV DNA in tissue samples is a gold standard for the detection of infection; however, the presence of viral DNA is often transient. The antibody response to HPV L1 virus-like particles (VLPs) remains relatively stable over time after clearance of infection, which predetermines it to be a useful epidemiologic marker that reflects cumulative exposure to HPV infection. Currently, two prophylactic HPV vaccines are commercially available, both containing antigens of the most prevalent HR HPV types 16 and $18 .^{8}$ One, in addition, contains also antigens of LR HPV types 6 and 11 . Both vaccines were shown to be effective in the protection against the development of diseases caused by the vaccine and closely related HPV types. The assessment of age-specific anti-HPV antibody prevalence allows for estimating and prospectively monitoring the impact of these vaccines as well as for planning the implementation of HPV vaccine into the cervical cancer prevention programmes in individual countries.

The aim of the study was to determine the prevalence of anti-HPV antibodies in the general population of Czech women and men. This is the first seroepidemiological study in the Czech Republic to assess the prevalence of HPV antibodies against four vaccine and two other closely related HR HPV types-HPV-31 and 33-in a broad range of ages of the Czech population.

\section{METHODS \\ Study population}

Serum samples originated from serological surveys performed in the Czech Republic by the National Institute of Public Health for multipurpose immunological surveys carried out since 1971. Persons enrolled in the survey were randomly selected in cooperation with the regional and district public health offices and general practitioners for adults, adolescents and children. Blood samples were 
collected in various urban and rural populations from healthy individuals 1-64 years old with no acute febrile disease and no signs of immunodeficiency on the sampling day. Each practitioner was provided by a list of blood samples to be taken by gender and age of the subjects. All individuals enrolled signed an informed consent form and in case of children, the informed consent form was signed by parents. All sera were aliquoted, registered in the Serum Bank of the National Institute of Public Health and kept frozen at $-20^{\circ} \mathrm{C}{ }^{9}$ The set of samples analysed in our study comprised sera collected in 1996 (\# 539, age span 6-20 years) and 2001 (\# 997, age span 9-64 years). In addition, we included sera from anonymous blood donors sampled between 1999 and 2005 (\# 1614), mandatorily tested for the presence of markers of infections transmitted by blood. All blood donors recruited in the study donated the blood free of charge. All subjects were divided based on gender and age. The differences in the prevalence of antibodies between various sets of samples are given in table 1 . There were no differences in the prevalence of HPV-specific antibodies in between the two cohorts of children 10-18 years old sampled in 1996 and 2001, as well as between the group of blood donors and serological survey 2001 in subjects up to 39 years of age when adjusted for age and gender. In the older age groups ( $\geq 40$ years), differences in the prevalence of HPV-specific antibodies for all tested antigens in between blood donors and serological survey participants has been found. Prevalences were two to three times higher in the group of blood donors, but the slope of the curve of prevalence by age was the same. Children and young individuals were categorised into 5 -year age groups to provide conclusive evidence for possible integration of HPV vaccination into the routine vaccination schedule in the Czech Republic.

\section{HPV serological test}

The presence of antibodies specific to HPV was tested using an in-house L1 VLP-based ELISA as described previously. ${ }^{10} 11$ HPV-6, $11,16,18,31$ and 33 VLPs were prepared in baculoviruses/insect cells expression system. Plates were coated with purified VLPs, blocked with bovine serum albumin and sera diluted 1:25 were incubated in duplicate. Antibodies bound were detected with donkey anti-human IgG, linked to horseradish peroxidise, the reaction was visualised by o-phenylenediamine and optical densities were read at 492 and $630 \mathrm{~nm}$. Control sera known to be positive and negative were tested on each plate. The optical density cut-off (CO) value was calculated separately for each antigen/plate. Each ELISA result was presented as a ratio between the absorbance of the sample and the respective $\mathrm{CO}$ value (OD index). To confirm the results, samples within $10 \%$ above the $\mathrm{CO}$ value as well as about one-quarter of all serum samples were retested. Only samples with repeated positive results were considered reactive.

\section{Statistical analysis}

Statistical calculations were performed using CDC EpiInfo TM software (V.3.5.1) and GraphPad Instat software (V.3.00). Test comparing the two groups was based on normal approximation of binomial distribution. The $\chi^{2}$ test with Yates' correction or Fisher's exact test was used to calculate ORs and 95\% CIs. The trend in seropositive rates across age categories was calculated by $\chi^{2}$ for trend. $p$ Values were two tailed, with $p<0.05$ considered statistically significant. In order to exclude the differences in age structure between the group of men and women, we used direct standardisation (WHO European standard). ${ }^{12}$

\section{RESULTS}

The overall prevalence of antibodies to HPV types 6, 11, 16, 18, 31 and 33 in 3150 subjects (age range $6-76$ years) were $21.2 \%$, $14.5 \%, 10.6 \%, 7.7 \%, 11.4 \%$ and $7.0 \%$, respectively. There were $25.8 \%$ seropositives for HPV-6 and/or 11, 15.0\% for HPV-16 and/ or $18,33.6 \%$ for any of the four vaccine types, $22.5 \%$ for any of the four HR HPV and $38.7 \%$ for any HPV type tested. The differences in the seropositivity rates between men and women are shown in table 2. In comparison to men, women had higher prevalence of antibodies specific for all antigens except for HPV11 VLPs. The differences were also found in case of seroprevalences of multiple HPV types in various combinations. All differences remained significant after standardisation for the WHO European age standard. The differences were more pronounced in the case of antibodies against HR HPV types.

The seroprevalence rates of antibodies specific to all HPV types tested were age dependent. The rate of seropositive women increased with age, peaking in the age group 25-29 years for HPV-6 (32.0\%), HPV-11 (19.7\%) and HPV-16 (21.2\%), in the age group 30-39 years for HPV-31 (23\%) and HPV-33 (15.3\%) and in the age group 50-59 years for HPV-18 $(16.7 \%)$. In men, the prevalence of antibodies reached the peak rates of $8.6 \%$ for HPV-33 in 25-29-year-olds, $18.0 \%$ for HPV-11 in 30-39-year-olds, 9.6\% for HPV-16, 6.9\% for HPV-18 and 8.3\% for HPV-31 in the age group 40-49 years and $31.8 \%$ for HPV-6 in subjects older than 59 years. The age-specific shifts in the seroprevalence rates were less marked in men, mainly in the case of antibodies to HR HPV types. The prevalence rates of antibodies to all antigens tested, except for HPV-11 (in both sexes), had a significant linear trend across all age categories.

The seropositivity rates of antibodies to HPV vaccine types (HPV-6, 11, 16 and 18) in women reached 27.0\% for HPV-16 and/or 18 and $50.0 \%$ for any of the four vaccine types at the age

Table 1 The differences in prevalences of HPV-specific antibodies between populations from serological surveys performed in 1996 and 2001 and between serological survey 2001 and blood donors

\begin{tabular}{|c|c|c|c|c|c|c|}
\hline & \multicolumn{6}{|l|}{ Age } \\
\hline & \multicolumn{2}{|c|}{$9-19$ years $(\mathrm{N}=539)^{*}$} & \multicolumn{2}{|c|}{$19-39$ years $(N=1419) \dagger$} & \multicolumn{2}{|c|}{$\geq 40$ years $(N=969) \dagger$} \\
\hline & $\overline{\text { OR }(95 \% \mathrm{CI}}$ & p Value & OR $(95 \% \mathrm{CI})$ & p Value & OR $(95 \% \mathrm{CI}$ & p Value \\
\hline HPV-11 & $1.5(0.9$ to 2.6$)$ & 0.140 & $1.1(0.8$ to 1.6$)$ & 0.388 & $0.4(0.3$ to 0.6$)$ & $>0.0001$ \\
\hline HPV-16 & $1.7(0.7$ to 3.9$)$ & 0.216 & $0.9(0.6$ to 1.2$)$ & 0.388 & $0.4(0.2$ to 0.6$)$ & $>0.0001$ \\
\hline HPV-18 & $1.1(0.5$ to 2.5$)$ & 0.877 & $0.8(0.5$ to 1.2$)$ & 0.225 & $0.2(0.1$ to 0.4$)$ & $>0.0001$ \\
\hline
\end{tabular}

Adjusted for age and gender.

*Comparison of samples from serological survey performed in 1996 and 2001.

†Comparison of samples from blood donors and serological survey 2001. 
Table 2 Seroprevalence rates for single and multiple human papillomavirus (HPV) types in women and men

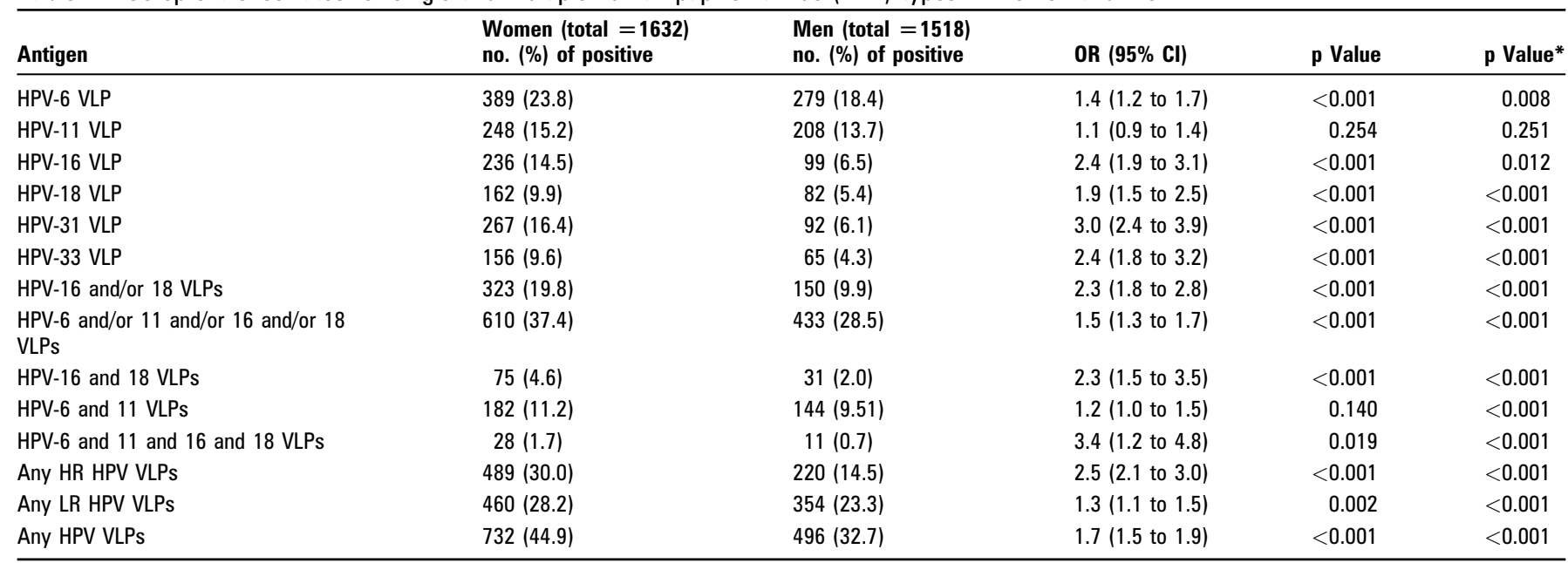

${ }^{*} p$ Value after standardisation on WHO European standard

HR, high risk; LR, low risk; VLP, virus-like particle.

of $30-39$. The seroprevalences for HPV-16 and/or 18 in men were increasing to $14.4 \%$ in 50-59-year-olds and for any of the four vaccine types to $37.6 \%$ in the oldest age group. The presence of antibodies to LR HPV-6 and/or HPV-11 was found in $28.2 \%$ of women, reaching a peak of $39.2 \%$ in the age group $30-39$ years, and in $23.3 \%$ of men, peaking with $31.8 \%$ in the oldest age group.

Seropositivity to any of the six HPV antigens tested was found in $44.9 \%$ of women and $32.7 \%$ of men, with the respective peaks of $59.9 \%$ in the age group $30-39$ years and $42.4 \%$ in over 59 -year-olds. As in the case of individual antigens, $p$ for trend in the combined seroprevalences across all age categories was statistically significant. Table 2 summarises the seroprevalence rates to multiple HPV types included in the currently available HPV vaccines and all HPV types tested in both women and men.

The presence of HPV-specific antibodies was detected in $16.5 \%$ (25/151) of children 6-9 years old. Mostly, the seropositivity for HPV-6 and/or 11 (13.9\%) was observed. Five (3.3\%) of these 6-9-year-old children possessed antibodies to HR HPV types.

\section{DISCUSSION}

This study is the largest (3150 subjects) population-based seroepidemiological study of the cross-sectional prevalence of antibodies to HPV performed in Central and Eastern Europe. To our knowledge, the only report on the HPV seroprevalence in this region are the study of Gorander et $a l^{13}$ analysing the seroprevalence of five HR HPV types in 199 blood donors in Katowice, Poland, and our study from $1998^{14}$ on the seroprevalence of HPV-1 and three HR HPV types in 450 serum samples from healthy subjects. Furthermore, to our knowledge, this is one of the two studies published to date showing the prevalence of antibodies specific for LR HPV-6 and -11 VLPs in children $<10$ years of age.

We have found high prevalence of antibodies to LR HPV types in children that reflects significant scale of non-sexual transmission in this population. Similar results were obtained in our previous study ${ }^{14}$ on substantially smaller cohort of subjects using type-common synthetic peptide derived from L2 protein of both HPV-6 and -11 . In children aged 6-9 years, the seroprevalence rates were $11.3 \%$ for HPV-6 and $10.6 \%$ for HPV-11. The seroprevalence for HR HPV types in this group of children was low comparable to our previous results and to those reported by others. ${ }^{15} 16$ The detailed analysis of the seropositivities in 1-year cohorts revealed in 11-, 12- and 13-years-old girls marked increase for HPV-6/HPV-11 from $6.1 \%$ to $16.7 \%$ and $25.9 \%$ and for HPV-16/HPV-18 from $0.0 \%$ to $6.7 \%$ and $14.8 \%$. In the same age cohorts of boys, there were $13.3 \%, 17.1 \%$ and 21.3\% HPV-6/11 seropositive, the seroprevalence for HPV-16/18 was low, with no clear trend. Though the main route of genital HPV transmission is sexual contact, markers of HPV infection have also been found in virgins (mostly for LR HPV, data not shown) and several studies suggest that the infection may be transmitted from mother to newborn before or during delivery and may persist for several years. ${ }^{17}$ It has also been shown that HPV infections in children might be acquired horizontally. ${ }^{18}$

This study has several limitations. As serum specimens analysed were originally collected in a scope of immunological surveys organised in the Czech Republic, the only information about age and sex was available. The same was true for blood samples from blood donors included in our study. Therefore, no demographic/behavioural data as well as information about previous/current HPV infection and diseases associated with $\mathrm{HPV}$, except for malignancies, were available for the analyses. Last but not least, the method we used in this study detects both neutralising and non-neutralising antibodies.

There are several factors affecting seroprevalence in the population: selection of the study cohort, demographic characteristics of the subjects enrolled such as the place of residenceurban versus rural-and the test used for the detection of antibodies. The last but not least factor is the assignment of the $\mathrm{CO}$ value above which the samples are considered reactive. Therefore, the comparison of the data across studies is difficult and should be made with caution. Nowadays, several assays are used for the detection of anti-HPV antibodies. Each of the assays measuring serum antibodies to VLP detects an overlapping but distinct set of antibody responses. ${ }^{19}$ The assay currently considered the gold standard for the assessment of HPVneutralising antibodies is an in vitro neutralisation assay. Specific neutralising antibodies prevent the infectious pseudovirions to enter the target cell. ${ }^{20}$ However, this assay is time consuming and not suitable for large clinical studies. The direct ELISA test with purified VLPs as antigens used in this study identifies neutralising and non-neutralising polyclonal antibodies, directed to various antigenic epitopes on the surface of VLP. ${ }^{21}$ Two other methods, multiplexed immunoassays, are based on 
antigens bound on Luminex microspheres- a competitive test with VLPs as antigen ${ }^{22}$ and a direct test with bacterially expressed L1 proteins/capsomeres. ${ }^{23}$

In agreement with other studies, ${ }^{24-26}$ we have found higher prevalence of HPV antibodies among women than among men for any of the HPV types tested. This observation is in contradiction with the reported higher prevalence of HPV DNA in men than in women. ${ }^{27} 28$ The lower prevalence of HPV-specific antibodies in men most likely reflects the differences in the site of HPV infection resulting in the difference in the immune response to HPV infection between genders. While in women, the infection occurs in the mucosal epithelium and the anatomy of the site of infection might contribute to long-term persistence of the virus, in men, the site of virus entry is probably the external keratinised tissue that is immunologically less prominent.

The seroprevalence rates for HPV-specific capsid antigens are age dependent. In women, the rate of seropositives peaked mainly in the age category of 30-39 years. The peak of prevalence of HPV-specific antibodies in men was observed later than in women -in the age group 40-49 years. Similar observations of the maximal seroprevalence shift among women and men were reported by others. ${ }^{24} 25$ The growing seropositivity in young individuals probably reflects repeated HPV infection early after the onset of sexual life. The rapid HPV acquisition after the sexual debut has been demonstrated in several prospective studies. $^{29} 30$ The decline in seropositivity in older age groups might be explained by a cohort effect, as older subjects had fewer lifetime sexual partners compared with younger ones. Non-negligible may be the difference between sexual behaviour of older rural and urban populations. Furthermore, also the effect of immune senescence in older age is to be considered.

The seroprevalence rates for any of four HPV vaccine types reached in women maximum in the age group of 30-39 years and in men in over 50-year-olds. Antibodies to HPV-16 and/or HPV-18 possessed $18.8 \%$ of women and $9.8 \%$ of men. The prevalence of antibodies to both HPV-16 and HPV-18 was rare in both genders. These findings are similar to the data reported in recent Australian and American studies on the healthy population, ${ }^{24} 25$ using competitive multiplexed assay, even though the rates of seropositives were lower. The differences in seroprevalence rates might be explained by different methods used.

We conclude that in order to prevent the diseases aetiologically linked with the vaccine HPV types, the routine vaccination against HPV in the Czech Republic should be implemented in girls under 13 years of age. Despite the increasing prevalence of antibodies to the HR HPV vaccine types in women over 15 years

\section{Key messages}

- The high prevalence of antibodies to low-risk HPV types in children suggests non-sexual transmission.

- Standardised prevalence of HPV antibodies differ between females and males.

- The prevalences of antibodies for various HPV types for both genders are age dependent.

- An increase of seropositives in group of 11 to 20-year-old girls supports the implementation of routine vaccination in age of $11-13$ years. of age, the catch-up vaccination of older/sexually active women will also be beneficial since simultaneous presence of antibodies specific to all vaccination types is rare.

Contributors EH participated in the design and coordination of the study, evaluated results, performed statistical analysis and drafted the manuscript. VL prepared antigens for assessment of antibody presence. JS performed the immunoassays. RT participated in the design of the study and helped to draft the manuscript. All authors read and approved the final manuscript.

Funding The work was funded by the Internal Grant Agency of the Ministry of Health of the Czech Republic, grants Nos. NS/9984 and NT/12372. The funder provided financial support for this project. The funder had no role in study design, data collection and analysis, decision to publish or preparation of the manuscript.

Competing interests RT is a member of the Czech GSK advisory board; the other authors have no conflicts of interest.

Patient consent Serum samples were derived from serological surveys performed in the Czech Republic by the National Institute of Public Health aimed to provide information about the immunity status of the population and basic data for decision on potential changes in the vaccination strategies. Such surveys have been carried out in the Czech Republic since 1971. Persons enrolled in the survey were randomly selected in cooperation with regional and district sanitary offices and with practitioners for adults, adolescents and children. Blood samples were collected in various urban and rural populations from healthy individuals $6-64$ years of age. The blood samples were taken from subjects with no acute febrile disease and no signs of immunodeficiency on the day of sampling. All enrolled subjects signed informed consent, in case of children, the informed consent was signed by parents. The blood sample was coded with regard to region, district, physician and client. All sera were aliquoted, registered in the Serum Bank of the Centre for Epidemiology and Microbiology, National Institute of Public Health, and kept frozen. The set of samples analysed in our study comprised of sera collected in the year 1996 and 2001. For the purpose of our study, all sera were marked by laboratory ID and no identification of particular person was possible. In addition, we included sera from anonymous blood donors sampled in the period of 1999-2005. All blood donors sign information consent form under the blood sampling. Those samples are mandatory tested for the presence of markers of infections transmitted by blood and discarded.

Ethics approval The study was approved by the Ethics committee of Institute of Hematology and Blood Transfusion (\#EK 2IGA/9/2009).

Provenance and peer review Not commissioned; externally peer reviewed.

Open Access This is an Open Access article distributed in accordance with the Creative Commons Attribution Non Commercial (CC BY-NC 3.0) license, which permits others to distribute, remix, adapt, build upon this work non-commercially, and license their derivative works on different terms, provided the original work is properly cited and the use is non-commercial. See: http://creativecommons.org/ licenses/by-nc/3.0/

\section{REFERENCES}

1. Arbyn M, Castellsague $X$, de Sanjose $S$, et al. Worldwide burden of cervical cancer in 2008. Ann Oncol 2011;22:2675-86.

2. Bouvard V, Baan R, Straif K, et al; WHO International Agency for Research on Cancer Monograph Working Group. A review of human carcinogens-Part B: biological agents. Lancet Oncol 2009;10:321-2.

3. de Sanjose S, Quint WG, Alemany $L$, et al; Retrospective International Survey and HPV Time Trends Study Group. Human papillomavirus genotype attribution in invasive cervical cancer: a retrospective cross-sectional worldwide study. Lancet Oncol 2010;11:1048-56.

4. Giuliano AR, Tortolero-Luna G, Ferrer E, et al. Epidemiology of human papillomavirus infection in men, cancers other than cervical and benign conditions. Vaccine 2008;26 (Suppl 10):K17-28.

5. Lacey CJ, Lowndes CM, Shah KV. Chapter 4: Burden and management of noncancerous HPV-related conditions: HPV-6/11 disease. Vaccine 2006;24(Suppl 3): S35-41.

6. Ho GY, Studentsov YY, Bierman R, et al. Natural history of human papillomavirus type 16 virus-like particle antibodies in young women. Cancer Epidemiol Biomarkers Prev 2004:13:110-16.

7. Safaeian M, Porras C, Schiffman M, et al; Costa Rican Vaccine Trial Group. Epidemiological study of anti-HPV16/18 seropositivity and subsequent risk of HPV16 and -18 infections. J Natl Cancer Inst 2010;102:1653-62.

8. Harper DM. Currently approved prophylactic HPV vaccines. Expert Rev Vaccines 2009:8:1663-79.

9. Kriz B. Multipurpose serological survey-its philosophy and objectives in the Czech Republic. Cent Eur J Public Health 2003;11:S4-6.

10. Smith EM, Ritchie JM, Pawlita M, et al. Human papillomavirus seropositivity and risks of head and neck cancer. Int J Cancer 2007;120:825-32.

11. Hrbacek J, Urban M, Hamsikova $E$, et al. Serum antibodies against genitourinary infectious agents in prostate cancer and benign prostate hyperplasia patients: a case-control study. BMC Cancer 2011;11:53. 
12. Standardization methods. In: Gail MH, Benichou J, eds. Encyclopedia of Epidemiologic Methods. 1st edn. Chichester: John Wiley \& Sons Ltd, 2000:871-83

13. Gorander S, Lagergard T, Romanik M, et al. Seroprevalences of herpes simplex virus type 2, five oncogenic human papillomaviruses, and Chlamydia trachomatis in Katowice, Poland. Clin Vaccine Immunol 2008;15:675-80.

14. Hamsikova $\mathbf{E}$, Ludvíková V, Smahel M, et al. Prevalence of antibodies to human papillomaviruses in the general population of the Czech Republic. Int $J$ Cancer 1998;77:689-94.

15. Marais DJ, Rose RC, Lane $C$, et al. Seroresponses to human papillomavirus types $16,18,31,33$, and 45 virus-like particles in South African women with cervica cancer and cervical intraepithelial neoplasia. J Med Virol 2000;60:403-10.

16. Dunne EF, Karem KL, Sternberg MR, et al. Seroprevalence of human papillomavirus type 16 in children. J Infect Dis 2005;191:1817-19.

17. Cason J, Mant CA. High-risk mucosal human papillomavirus infections during infancy \& childhood. J Clin Virol 2005;32(Suppl 1):S52-8.

18. Syrjanen S. Current concepts on human papillomavirus infections in children. APMIS 2010;118:494-509.

19. Schiller JT, Lowy DR. Immunogenicity testing in human papillomavirus virus-likeparticle vaccine trials. J Infect Dis 2009;200:166-71.

20. Steele J, Collins S, Wen K, et al. Measurement of the humoral immune response following an incident human papillomavirus type 16 or 18 infection in young women by a pseudovirion-based neutralizing antibody assay. Clin Vaccine Immunol 2008;15:1387-90.

21. Vaccarella S, Franceschi S, Clifford GM, et al. Seroprevalence of antibodies against human papillomavirus (HPV) types 16 and 18 in four continents: the International Agency for Research on cancer HPV prevalence surveys. Cancer Epidemiol Biomarkers Prev 2010;19:2379-88.
22. Opalka D, Lachman CE, MacMullen SA, et al. Simultaneous quantitation of antibodies to neutralizing epitopes on virus-like particles for human papillomavirus types 6,11 , 16, and 18 by a multiplexed luminex assay. Clin Diagn Lab Immunol 2003:10:108-15.

23. Syrjanen S, Waterboer T, Sarkola M, et al. Dynamics of human papillomavirus serology in women followed up for 36 months after pregnancy. J Gen Virol 2009:90:1515-26.

24. Newall AT, Brotherton JM, Quinn HE, et al. Population seroprevalence of human papillomavirus types $6,11,16$, and 18 in men, women, and children in Australia. Clin Infect Dis 2008:46:1647-55.

25. Markowitz LE, Sternberg M, Dunne EF, et al. Seroprevalence of human papillomavirus types 6, 11, 16, and 18 in the United States: National health and Nutrition Examination survey 2003-2004. J Infect Dis 2009:200:1059-67.

26. Michael KM, Waterboer T, Sehr P, et al. Seroprevalence of 34 human papillomavirus types in the German general population. PLoS Pathog 2008;4: e1000091.

27. Giuliano AR, Lazcano-Ponce E, Villa LL, et al. The human papillomavirus infection in men study: human papillomavirus prevalence and type distribution among men residing in Brazil, Mexico, and the United States. Cancer Epidemiol Biomarkers Prev 2008;17:2036-43.

28. Vardas $\mathbf{E}$, Giuliano AR, Goldstone $\mathrm{S}$, et al. External genital human papillomavirus prevalence and associated factors among heterosexual men on 5 continents. $J$ Infect Dis 2011;203:58-65.

29. Winer RL, Feng 0 , Hughes JP, et al. Risk of female human papillomavirus acquisition associated with first male sex partner. J Infect Dis 2008;197:279-82.

30. Munoz N, Mendez F, Posso H, et al; Instituto Nacional de Cancerologia HPV Study Group. Incidence, duration, and determinants of cervical human papillomavirus infection in a cohort of Colombian women with normal cytological results. J Infect Dis 2004:190:2077-87. 